\title{
In situ structure characterization of perovskite-based catalysts
}

\author{
S. Gallus, E. Budiyanto, C. Weidenthaler \\ Max-Planck-Institut für Kohlenforschung, Kaiser-Wilhelm-Platz 1, 45470 Mülheim an der Ruhr \\ weidenthaler@mpi-muelheim.mpg.de
}

Catalytic processes are indispensable in energy research. It is essential to enhance their environmental friendliness and resource-efficiency by exploring and optimizing new reaction routes for future sustainability. One major topic in this context is the chemical energy storage using eg. hydrogen, in a pure state or as hydrogen-carrier molecules. The release of hydrogen from carrier molecules, such as ammonia $\left(\mathrm{NH}_{3}\right)$, is achieved by a catalytic reaction. Among others, promising catalysts for ammonia decomposition are nickel-based catalysts [1]. Perovskites, eg. $\mathrm{LaNiO}_{3}$, can serve as catalyst precursors.

The synthesis of $\mathrm{LaNiO}_{3}$ was conducted via incipient wetness impregnation of mesoporous carbon spheres and subsequent sintering [2]. Detailed microstructure analysis was performed via transmission electron microscopy that enabled the identification of Ruddlesden-Popper faults. These [LaO]-[LaO] shear faults have been reported in the past to be present in $\mathrm{LaNiO}_{3}$ thin films grown on various substrates [3,4]. With this information, a Rietveld refinement of synchrotron powder diffraction data could be performed using a stacking fault model based on the superstructure, space group Pm-3m, of the original $\mathrm{LaNiO}_{3}$ crystal structure, space group R-3cH (Fig. 1 a). In addition, the local structure of the perovskite was investigated by total scattering neutron and synchrotron experiments and subsequent pair distribution function analysis.

The aim of this work is the correlation of structure properties with the catalytic performance of perovskites with different chemical compositions. The catalytic performance of the synthesized material was tested during $\mathrm{NH}_{3}$ cracking experiments. For $\mathrm{LaNiO}_{3}$, a conversion of $70 \%$ could be achieved at $550^{\circ} \mathrm{C}$ with a gas flow of $15000 \mathrm{ml} / \mathrm{g}^{-1} \mathrm{~h}^{-1}$ of $100 \% \mathrm{NH}_{3}$. The structural behavior during the reaction was investigated by in situ synchrotron diffraction experiments at the high-resolution powder diffraction beamline P02.1 (PETRA III, DESY). A decomposition of the perovskite via intermediate states and the reduction to the active phase of metallic Ni on $\mathrm{La}_{2} \mathrm{O}_{3}$ could be observed (Fig. 1 b).
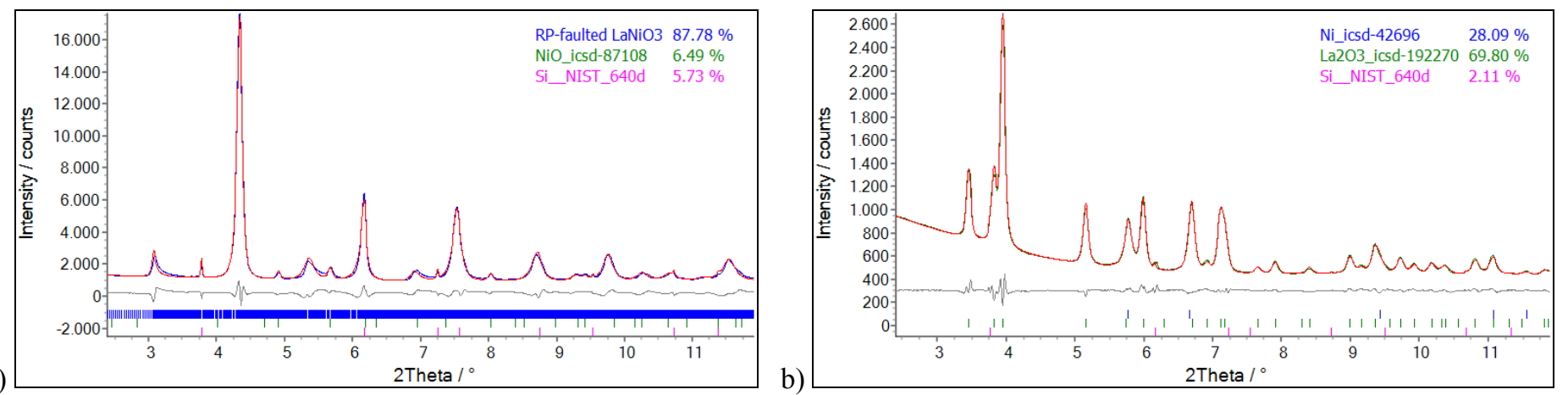

Figure 1. a) Rietveld refinement plot of $\mathrm{LaNiO}_{3}$ with a crystal structure model containing Ruddlesden-Popper-type faults stacked along the $c$-axis, $a=b=3.8517(3) \AA, \alpha=\beta=\gamma=90^{\circ}$, space group P1, by-phases are Si and NiO; b) Rietveld refinement plot of the activated catalyst $\left(\mathrm{Ni}\right.$ on $\left.\mathrm{La}_{2} \mathrm{O}_{3}\right)$ obtained in situ during the ammonia decomposition experiment. Si was added as an internal standard.

[1] Bell, T. E. \& Torrente-Murciano, L. (2016). Top. Catal., 59, 1438.

[2] Schwickardi, M., Johann, T., Schmidt, W. \& Schüth, F. (2002). Chem. Mat. 14, 3913.

[3] Bak, J., Bae, H. B., Kim, J., Oh, J. \& Chung, S.-Y. (2017). Nano Lett. 17, 3126.

[4] Bak, J., Bae, H. B., Oh, C., Son, J. \& Chung, S.-Y. (2020). J. Phys. Chem. Lett. 11, 7253.

\section{Keywords: perovskites; microstructure; stacking faults; ammonia decomposition; catalysis}

We acknowledge DESY (Hamburg, Germany) a member of the Helmholtz Association HGF, for the provision of experimental facilities. Parts of this research were carried out at PETRA III, beamline P02.1. We further acknowledge the Science and Technology Facilities Council (STFC) for access to neutron beamtime at the ISIS Neutron and Muon Source at the General Materials Diffractometer (GEM) instrument in the Materials Characterisation Laboratory.

We thank Hilke Petersen, Jan Ternieden, Jo-Chi Tseng, Claudio Pistidda, Giovanni Capurso, and David Keen for their support in conducting the experiments during the beamtimes. 